\title{
Propuesta de un diseño de investigación mixto para el estudio del ritmo en diferentes variedades dialectales del español
}

\author{
Karina Cerda-Oñate* \\ Pontificia Universidad Católica de Chile, Chile
}

\section{INTRODUCCIÓN}

El estudio de la prosodia de las lenguas ha avanzado a pasos agigantados en los últimos 40 o 30 años en la lingüística y, más específicamente, en la fonología y la fonética. Mucho ha contribuido la disponibilidad de softwares e instrumentos que han facilitado las mediciones de la frecuencia fundamental, así como también el análisis de la trayectoria y cambios en las curvas de entonación para hacer tipologías respecto de la melodía del habla a nivel fonético y la entonación a nivel fonológico y su relación con la sintaxis, por ejemplo.

Empero, no todas las materias de investigación han crecido de manera tan veloz en el estudio de la prosodia: el ritmo de las lenguas es claramente un objeto de estudio que pese a las innovaciones, es escasamente investigado, especialmente en el español. Si bien existen distinciones claras y asentadas en la comunidad lingüística acerca de los tipos de ritmos de las lenguas, basadas en las propuestas de Pike (1945), Abercrombie (1967) y Roach (1982), la mayoría de estas clasificaciones iniciales no se establecieron

* Para correspondencia, dirigirse a: Karina Cerda-Oñate (kecerda@uc.cl), Departamento de Ciencias del Lenguaje, Facultad de Letras, Pontificia Universidad Católica de Chile, Campus San Joaquín, Av. Vicuña Mackenna 4860, Macul, 7820436, Santiago, Chile. 
en base a datos empíricos ni tampoco prentendían entregar explicaciones respecto a todos los fenómenos lingüísticos que entran en juego durante la producción y percepción del ritmo de una lengua o dialecto específico.

A este respecto, cabe señalar que clásicamente se distinguen dos tipos de compases rítmicos para las lenguas naturales: ritmo acentual y ritmo silábico (Pike 1945; Abercrombie 1967; Roach 1982). Sin embargo, esta clasificación tipológica es considerada oscura por los mismos investigadores del ritmo del habla, pues no clarifica qué lenguas pertenecen a qué categorías ni toma en consideración las variedades dialectales de las lenguas naturales.

En 1983, Dauer hizo una revisión sobre las nociones clásicas del ritmo en las lenguas naturales. A partir de esto, Ramus, Nespor y Mehler (1999) y Low, Grabe y Nolan (2000) propusieron nuevas formas de medición del ritmo, con el objetivo de explicar cómo funcionaba la producción y percepción de este fenómeno en las lenguas naturales. En su momento, ambos grupos de investigadores indicaron que, al parecer, el ritmo de las lenguas se relacionaba más bien con la duración intervocálica que con la duración silábica, entendiendo que las vocales son normalmente núcleo de sílaba y, que, por tanto, las unidades intervocálicas serían las que guiarían el compás de una lengua.

Básicamente, lo que proponían estos autores es que las lenguas no girarían en torno al pie silábico (ritmo acentual) o a la sílaba (ritmo silábico), sino que más bien aquello que producimos y percibimos, que ha sido denominado ritmo silábico o acentual, consiste en el hecho de que en algunas lenguas los segmentos intervocálicos tienen una duración y una frecuencia de aparición similar y, en cambio, en otras lenguas, los segmentos intervocálicos tendrían una duración dispar y una frecuencia menor de aparición.

\section{BREVE REVISIÓN SOBRE EL ESTUDIO DEL RITMO EN LAS LENGUAS NATURALES Y SUS VARIEDADES DIALECTALES}

En virtud de los objetivos de esta nota, se presentará una breve revisión del concepto de ritmo y su estudio en las lenguas naturales y sus variedades dialectales, con el objetivo de enunciar el objeto de estudio en cuestión. En primer lugar, y con respecto al concepto de ritmo, cabe destacar que tradicionalmente se ha hablado de dos tipos de ritmos que diferencian a las lenguas naturales: el ritmo silábico, isocronía silábica o compás silábico y el ritmo acentual o isocronía acentual (Pike 1945; Abercrombie 1967). 
El primer tipo, el ritmo silábico, es un tipo de ritmo que en principio estaría gobernado por la estructura silábica de la lengua; es decir, el ritmo de la lengua se desplegaría en torno a la sílaba y no se buscaría cumplir con un compás similar entre las diferentes sílabas de la lengua, sino que más bien mantener la estructura silábica de la lengua específica (Pike 1945; Abercrombie 1967). En cambio, en el caso del ritmo acentual, la estructura rítmica de la lengua giraría en torno al pie acentual, es decir, "[...] el lapso temporal entre una sílaba acentuada y la siguiente sílaba acentuada tenderá a ser constante" (Toledo 2010: 94). Este tipo de ordenamiento permitiría generar distancias temporales similares en la lengua, a pesar de que exista un número de sílabas diferentes en los distintos enunciados. Entre las exponentes de las lenguas con ritmo silábico se encontrarían el español, el francés, el turco, entre otras; por su parte, las lenguas germánicas, como el inglés, el alemán y el holandés tendrían un ritmo de tipo acentual. (Abercrombie).

Ahora, si bien estas categorías fueron establecidas por Pike (1945) y posteriormente reafirmadas por Abercrombie (1967) y Roach (1982), ninguno de los tres autores entregó suficiente evidencia empírica para establecer las distinciones antes mencionadas más allá de algunos datos acerca de la duración silábica. Por esta misma razón, en 1999 Ramus, Nespor y Mehler propusieron tres índices para medir la variación de los segmentos intervocálicos e interconsonánticos de las lenguas, con el fin de poder discriminar de manera cuantitativa los tipos rítmicos de las lenguas naturales en corpus extensos. Posteriormente, y tomando como punto de partida el trabajo de Ramus et al. (1999), Low et al. (2000) modificaron los índices propuestos por estos autores y postularon el índice Pairwise Variability Index, que se explicará en la próxima sección.

\section{MÉTODOS CUANTITATIVOS UTILIZADOS PARA MEDIR EL RITMO}

En relación con lo anterior, a continuación se discutirán dos mediciones de tipo cuantitativo que son utilizadas actualmente para medir el ritmo en las lenguas naturales y sus variedades dialectales: en primer lugar, se describirá la propuesta de Ramus et al. (1999) y, posteriormente, se explicará la propuesta de Low et al. (2000).

Ramus et al. (1999) propusieron, a partir de un corpus en el que separaron segmentos vocálicos y consonánticos (las glides prevocálicas se etiquetaron 
como consonantes y las glides postvocálicas se categorizaron como vocales), hacer mediciones en torno a los intervalos vocálicos y consonánticos, que definieron en virtud del ataque y la coda de cada segmento; es decir, un intervalo vocálico o consonántico comienza con el ataque de la vocal o consonante y termina con la coda de la vocal o consonante.

A partir de esta unidad de medida, los autores postularon tres tipos de índices de medición. El primer índice, denominado \% V, establece la proporción de intervalos vocálicos en cada enunciado; es decir, la suma de intervalos vocálicos dividida por la duración total del enunciado. El segundo índice mide la desviación estándar de la duración de los intervalos vocálicos en cada enunciado $(\Delta \mathrm{V})$; y, el tercero calcula la desviación estándar de la duración de los intervalos consonánticos en los enunciados $(\Delta \mathrm{C})$.

Respecto de esto, los autores comentan que los índices más productivos para la medición del ritmo en las lenguas naturales son $\% \mathrm{~V}$, que da cuenta de los intervalos vocálicos divididos por la duración total del enunciado, y el índice $\Delta C$, que corresponde a la desviación estándar de los intervalos consonánticos. Con respecto al índice $\Delta \mathrm{V}$, los autores indicaron que este no parece ser tan productivo como medición del ritmo en las lenguas naturales, pues existen fenómenos particulares en algunas lenguas, como la reducción vocálica, el alargamiento vocálico, etc., que pueden interferir con la medición.

Por su parte, Low et al. (2000) propusieron el índice Pairwise Variability Index (desde ahora PVI) para medir el ritmo en las lenguas naturales. Este índice tenía como objetivo dar cuenta del ritmo tomando en consideración fenómenos como la reducción vocálica o el alargamiento vocálico, por ejemplo. Básicamente, el índice formulado por estos autores consiste en calcular la diferencia de duración entre sílabas sucesivas (par silábico) por medio del cálculo del valor absoluto de la diferencia y, posteriormente, dividirlo por el promedio de duración del par en cuestión. Luego, las diferencias de los pares se suman y se dividen por la diferencia entre los pares silábicos. Finalmente, este resultado es multiplicado por 100, dado que la normalización previa produce resultados fraccionarios. De acuerdo con Low et al., al realizar las operaciones antes mencionadas, se normalizan los valores obtenidos, pues se suprime la variación intersujeto derivada de la velocidad de habla. 
Figura 1. Fórmula para calcular el índice Pairwise Variability Index

$$
\begin{aligned}
& \text { PVI }=100 \times\left[\sum_{k=1}^{m-1}\left|\frac{d_{k}-d_{k+1}}{\left(d_{k}+d_{k+1}\right) / 2}\right| /(m-1)\right] \\
& \text { where } m=\text { number of vowels in utterance } \\
& d=\text { duration of the } k^{\text {th }} \text { vowel }
\end{aligned}
$$

Fuente. Low Ee-Ling, Esther Grabe y Francis Nolan. 2000. Quantitative Characterizations of Speech Rhythm: Syllable-Timing in Singapore English. Language and speech 43(4): 377-401

Con respecto a estos dos métodos, y a modo de resumen, se puede apreciar que ambos grupos de investigadores proponen mediciones de tipo cuantitativo que toman en consideración unidades distintas, como la sílaba, los intervalos consonánticos, los intervalos vocálicos y la duración de los enunciados. En este sentido, es plausible señalar que estos dos métodos no son excluyentes, pues tienen como objetivo medir el ritmo en torno a unidades diferentes. De este modo, se propone que es conveniente trabajar con ambas mediciones, en tanto entregan datos productivos con respecto a unidades como los intervalos vocálicos y consonánticos, así como también acerca de la sílaba.

\section{ALGUNOS ESTUDIOS QUE HAN UTILIZADO LOS ÍNDICES DE RAMUS ET AL. (1999) Y/O LOW ET AL. (2000)}

A continuación, se hará una breve revisión de trabajos que utilizan los índices propuestos por Ramus et al. (1999) y/o por Low et al. (2000). En primer lugar, se discutirán algunos trabajos sobre diferentes variedades dialectales del español y, posteriormente, se reseñarán investigaciones sobre distintas variedades dialectales del inglés.

En el español, Toledo (2010) trabajó tanto con los índices propuestos por Ramus et al. (1999) como con los formulados por Low et al. (2000). En particular, Toledo analizó un corpus de tres muestras del proyecto AmperEspaña. Las muestras pertenecían a los proyectos Amper de Aragón, Canarias y Granada. El corpus se analizó en virtud de la duración de los segmentos de intervalos vocálicos y consonánticos, de acuerdo con lo propuesto por Ramus et al. y Low et al. Los resultados obtenidos por Toledo (2010), evidenciaron que el español sería una lengua de ritmo silábico, reafirmando lo propuesto 
por Pike (1945) y Abercrombie (1967). Ahora bien, pese a sus resultados, el autor indica en su artículo la necesidad de tomar en cuenta que el ritmo es una categoría sensible a cambios dialectales y sociolectales (Toledo: 107), algo que discutiremos más adelante en esta nota.

Por su parte, Prieto, Vanrell, Astruc, Payne y Post (2012) analizaron un corpus de 720 enunciados, obtenidos de un corpus de lectura de 24 hablantes femeninos de entre 28 a 49 años. Específicamente, las participantes eran ocho hablantes de inglés británico estándar de la zona de Cambridge, ocho hablantes de español peninsular central de la zona de Madrid y ocho hablantes de catalán central de la zona de Barcelona. Las colaboradoras leyeron 10 enunciados en 3 tareas experimentales distintas, es decir, 30 enunciados en total, respectivamente. El corpus obtenido fue segmentado, analizado silábicamente y prosódicamente. Una vez realizada esta acción, Prieto et al. extrajeron los intervalos vocálicos y consonánticos y aplicaron las siguientes mediciones de ritmo: \%V, $\Delta \mathrm{V}$ y $\Delta \mathrm{C}$ (Ramus et al. 1999), nPVI-V y rPVI-V (Low et al. 2000), VarcoC y VarcoV (Dellwo 2006 en Prieto et al. 2012) y Ferragne y Pellegrino (2004 en Prieto et al., 2012).

Respecto de los resultados, Prieto et al. (2012) encontraron que las mediciones de tipo vocálico, tales como $\% \mathrm{~V}, \Delta \mathrm{V}$, VarcoV y nPVI-V entregaban un índice más robusto de las clases rítmicas que las mediciones de tipo consonántico. Con relación a esto, Prieto et al. (2012) indicaron que las mediciones de tipo consonántico, tales como $\Delta \mathrm{C}$ y VarcoC serían más sensibles a diferencias fonotácticas que a diferencias de tipo rítmico. Por otra parte, las autoras también observaron que todos los índices dejaban en claro una diferencia sistemática entre la clase rítmica del inglés y la clase rítmica del catalán y el español.

También, De Pinho (2013) analizó las variedades dialectales del español de Chile y España y, al igual que Toledo (2010) y Prieto et al. (2012), utilizó los índices propuestos por Low et al. (2000) para medir la duración intervocálica. En cuanto a la muestra, De Pinho trabajó con un corpus de dos enunciados extraídos de noticiarios televisivos de Chile y España. Los resultados de De Pinho fueron claros en relación con los valores obtenidos en los tres índices que analizó: tanto el español de Chile como el español de España serían lenguas de ritmo silábico.

Para el inglés de Nueva Zelanda, Nokes y Hayes (2012) también seleccionaron las mediciones propuestas por Low et al. (2000). Específicamente, Nokes y Hayes realizaron un estudio de tipo longitudinal sobre el inglés de Nueva Zelanda y trabajaron con un corpus de un millón seiscientos mil segmentos vocálicos, el cual provenía de las grabaciones de 506 participantes. La investigación arrojó como resultado el hallazgo de un proceso de reducción de la duración vocálica en el inglés de Nueva Zelanda, 
de acuerdo con el índice nPVI. En otras palabras, la investigación demostró un cambio en el inventario vocálico del inglés de Nueva Zelanda a partir de las mediciones propuestas por Low et al. (2000).

Finalmente, Tan y Low (2014) analizaron el ritmo del inglés de Malasia en comparación con el ritmo del inglés de Singapur. Las investigadoras analizaron muestras de habla en situación de lectura y de habla espontánea de 10 hablantes de inglés de Malasia y las compararon con muestras de habla en situación de lectura y habla espontánea de 10 hablantes de inglés de Singapur. Para el análisis, Tan y Low utilizaron los índices propuestos por Low et al. (2000) y el índice VarcoV (White y Mattys 2007) para medir la duración vocálica.

Los resultados de Tan y Low (2014) indicaron que el inglés de Singapur exhibe más cualidades de una lengua de ritmo acentual que el inglés de Malasia, tanto a nivel de lectura como a nivel de conversación espontánea. En cuanto a la duración vocálica, Tan y Low no adviertieron diferencias significativas entre ambos dialectos del inglés. Finalmente, Tan y Low mencionaron que si bien los hallazgos son interesantes, sería pertinente incluir una tarea perceptual en el futuro para evaluar el ritmo de ambos dialectos.

A modo de resumen de esta breve revisión bibliográfica, cabe señalar que en la mayoría de los artículos revisados se observa una tensión respecto del concepto de ritmo en las lenguas naturales. En este sentido, se aprecia que si bien existen categorías binarias que se han utilizado tradicionalmente para referirse al ritmo en las lenguas naturales, tales como ritmo silábico y acentual (Pike 1945; Abercrombie 1967), se evidencia también una escasez respecto de datos obtenidos en base a mediciones de tipo empírico, que sean capaces de constatar que las regularidades indicadas por Pike y Abercrombie se cristalizan realmente en las lenguas naturales.

\section{REVISIÓN SOBRE PRINCIPIOS BÁSICOS DE LOS MÉTODOS ETNOGRÁFICOS Y SU APLICABILIDAD AL ESTUDIO DEL RITMO EN LAS LENGUAS NATURALES Y SUS VARIEDADES DIALECTALES}

Para comenzar a hablar de la aplicabilidad de los métodos etnográficos al estudio del ritmo, se hará una revisión sobre los principios básicos del método etnográfico y, paralelamente, se discutirá la pertinencia de la aplicación de estos métodos al estudio del ritmo en las lenguas naturales. 
Antes de todo, es importante definir en qué consiste la etnografía como método de estudio. De acuerdo con Rodríguez Gómez, Gil Flores y García Jiménez (1996) la etnografía es "[...] el método de investigación por el que se aprende el modo de vida de la unidad social. A través de la etnografía se persigue la descripción y reconstrucción analítica de carácter interpretativo de la cultura, formas de vida y estructura social del grupo investigado. Pero también, bajo el concepto etnografía, nos referimos al producto del proceso de investigación: un escrito etnográfico o retrato del modo de vida de una unidad social" (21).

En relación con lo anterior, Sealey (2007) indica que la etnografía lingüística puede revelar detalladamente cómo los hablantes negocian las distintas restricciones y posibilidades de su experiencia situada particular. Así también, Blommaert (2005: 205 en Sealey 2007: 646) señala que en general existe consenso acerca de que la identidad de los sujetos se construye a través de prácticas que producen, permiten y manifiestan identidad; en este sentido, la identidad sería una suerte de identificación colectiva, un trabajo semiótico que proviene del condicionamiento social.

De este modo, la etnografía se relaciona con el estudio del ritmo en las lenguas naturales y sus variedades dialectales, pues el ritmo de una lengua es un fenómeno situado y coconstruido en comunidades de habla específicas, por ejemplo, la variedad dialectal del español de Chile. De esta manera, un estudio del ritmo que involucre métodos etnográficos, como por ejemplo, entrevistas, experiencias personales o historias de vida permitiría investigar el ritmo no solo de manera cuantitativa, sino también de manera cualitativa, en tanto un fenómeno que es compartido por una comunidad de habla y que está vinculado a su identidad y patrimonio cultural.

A continuación, se establecerán ciertos requisitos básicos de la etnografía de acuerdo con lo propuesto por Rodríguez Gómez et al. (1996) y se evaluarán uno por uno en virtud de su aplicabilidad al estudio del ritmo en las lenguas naturales y sus variedades dialectales.

Según los autores, el primer requisito básico de la etnografía es la observación directa. Rodríguez Gómez et al. (1996) indican que "[...] la primera obligación del etnógrafo es permanecer donde la acción tiene lugar y de tal forma que su presencia modifique lo menos posible tal acción" (21). Con respecto al estudio del ritmo en las lenguas naturales y sus variedades dialectales, es claro que la observación directa es menester a la hora de investigar un fenómeno lingüístico como el ritmo en las lenguas. De esta manera, es plausible señalar que este requisito de la metodología etnográfica es pertinente y adecuado para el objeto de estudio en cuestión.

Como segundo requisito, los autores establecen que la metodología etnográfica exige pasar tiempo suficiente en el escenario a investigar. A este 
respecto, los autores mencionan la dificultad de hacer etnografía cultural seria en un período de dos semanas, por ejemplo (Rodríguez Gómez et al. 1996: 21-22). Con referencia al estudio del ritmo en las lenguas naturales y sus variedades dialectales, este requisito puede ser o no ser pertinente en virtud del conocimiento que posea el investigador acerca de la variedad dialectal en cuestión. Por ejemplo, un investigador que forma parte de una comunidad de habla específica no requerirá pasar un año estudiando el ritmo del dialecto de su comunidad de habla, pues a este investigador las características suprasegmentales de esa variedad dialectal le son comunes $\mathrm{y}$, muy posiblemente, son parte de su propia habla.

El tercer punto que destacan los autores para realizar investigación en etnografía es contar con un gran volumen de datos registrados. En cuanto a esto, postulan que el papel del observador es fundamental al momento de recoger todo tipo de información, ya sea por medio de notas de campo u otros recursos tecnológicos disponibles. Así también, los autores argumentan que los etnógrafos deben ser buenos recopiladores de artefactos, productos y documentos (Rodríguez Gómez et al. 1996: 22). Respecto a la investigación del ritmo en las lenguas naturales y sus variedades dialectales, es claro que los datos, ya sean de naturaleza cuantitativa o cualitativa, enriquecen la investigación del ritmo en las lenguas. Por ende, este requisito de los métodos etnográficos también es pertinente y apropiado para la investigación del ritmo en las lenguas naturales y sus variedades dialectales.

Rodríguez Gómez et al. (1996) juzgan que una cuarta condición a considerar al hacer investigación utilizando un método etnográfico es el carácter evolutivo de este tipo de investigaciones. En lo relativo a esto, los autores señalan que, por lo menos, al inicio de sus trabajos, los etnógrafos no deberían trabajar con hipótesis específicas, sino que deberían comenzar sus investigaciones con una perspectiva totalmente abierta, para de esta forma encontrar el mayor número de relaciones posibles. En lo concerniente a esta condición para el estudio del ritmo, esta no supone un problema para este tipo de estudios desde una perspectiva tipológica. De hecho, el modelo que se propone en esta nota no parte de una premisa particular, sino que más bien busca describir y tipologizar los distintos sistemas rítmicos de las variedades dialectales de las lenguas naturales a partir de datos cuantitativos y cualitativos.

La quinta condición que arguyen los autores respecto de los métodos etnográficos es la utilización de instrumentos. En relación con lo anterior, los autores mencionan que los instrumentos deben utilizarse solo cuando sean realmente importantes, pertinentes y exista una conciencia clara por parte del investigador sobre el enfoque y el contexto donde se sitúa el estudio (Rodríguez Gómez et al. 1996: 22). Esto se debe a que la cuantificación no 
es el punto de inicio ni el objetivo de utilizar métodos de tipo etnográfico, sino que más bien los instrumentos cuantitativos se consideran un refuerzo para las interpretaciones y/o para la comprobación de ciertos tipos de datos (Rodríguez Gómez et al.: 22).

Con respecto al punto anterior, es preciso hacer una reflexión mayor, dado que si bien se ha considerado en esta propuesta el uso de datos etnográficos con el objetivo de incorporar una perspectiva cualitativa al estudio del ritmo en las variedades dialectales de las lenguas naturales, es claro que en un diseño de tipo mixto, la cantidad de datos sí guarda relevancia en la recolección de datos cuantitativos. En lo relativo a esto entonces, es importante señalar que el fin y objetivo de utilizar métodos etnográficos en un diseño mixto no es el de la acumulación de datos, pero que, pese a esto, en esta investigación sí se aspirará a obtener la mayor cantidad de datos cuantitativos sobre el fenómeno en cuestión.

La sexta condición que postulan los autores se relaciona con el objeto de estudio de la etnografía. Respecto de este, subrayan que el objetivo principal de la etnografía es "[...] descubrir el conocimiento cultural que la gente guarda en sus mentes, cómo es empleado en la interacción social y las consecuencias de su empleo" (Rodríguez Gómez et al. 1996: 22). En lo que concierne a este punto, el estudio del ritmo de las lenguas resulta ser un objeto de estudio pertinente para la etnografía, en tanto conocimiento cultural de los sujetos de las comunidades de habla, así como también debido a que el ritmo es uno de los elementos suprasegmentales del habla, que es uno de los vehículos de la interacción social.

Finalmente, los autores establecen que es necesario tener una visión holística selectiva y hacer una contextualización particular a la hora de realizar una investigación de carácter etnográfico. Respecto del holismo, señalan que es un ideal deseable, pero que debe ser reducido operativamente al entorno investigativo. Es decir, si bien se puede tener una perspectiva holística, es necesario de todas maneras operacionalizar de cierta manera el entorno a investigar, con tal de que el fenómeno no desborde. Al respecto del holismo en esta investigación, es importante señalar que esta perspectiva puede ser pertinente en la medida en que sea operativa. Es decir, una visión holística selectiva, tal como postulan los autores, es relevante para el estudio del ritmo de las variedades dialectales de las lenguas, pero es menester que esta visión no entorpezca la investigación, en tanto el ritmo de por sí ya es un fenómeno multifactorial y complejo.

Por otra parte, Spindler y Spindler (1992 en Rodríguez Gómez et al. 1996: 23) proponen una serie de criterios para la investigación etnográfica, que serán también discutidos en relación con su pertinencia al objeto de estudio en cuestión. El primer criterio propuesto por los autores son las 
observaciones contextualizadas. En relación con el objeto de estudio, se estima que las observaciones contextualizadas son pertinentes y efectivas en el estudio del ritmo en las lenguas y sus variedades dialectales, en tanto fenómeno lingüístico situado. Un segundo criterio propuesto por Spindler y Spindler (1992 en Rodríguez Gómez et al. 1996: 23) es el de generación de hipótesis que emerjan in situ. En cuanto a este punto, cabe indicar que no es apropiado para el estudio en cuestión, pues, dado el carácter multifactorial del fenómeno, las hipótesis no pueden emerger in situ, sino que más bien se postulan una vez que se cuenta con datos de tipo cuantitativo y cualitativo.

El tercer criterio que sugieren los autores es la necesidad de observación prolongada y repetitiva. Con respecto a este punto, es posible argumentar que este criterio puede ser adecuado para el estudio del ritmo en las lenguas naturales y sus variedades dialectales solo si se considera como prolongada y repetitiva la observación de diferentes hablantes de una comunidad de habla particular. Así también, los autores mencionan como cuarto criterio incluir el punto de vista local por medio de las inferencias realizadas a partir de las observaciones y las diferentes formas de indagación etnográfica. En relación con esto, se juzga que es un criterio apropiado para la investigación del ritmo de las lenguas naturales y sus variedades dialectales, dado que se recolectarán datos cualitativos en las comunidades de habla específicas, con el objetivo de situar el fenómeno a nivel cultural e identitario.

El quinto punto mencionado por Spindler y Spindler (1992 en Rodríguez Gómez et al., 1996: 23) es la observación etnográfica de la conducta y comunicación social de los participantes, con el objetivo de obtener datos cualitativos sobre su conocimiento cultural. Si bien este punto no es necesariamente del todo pertinente para la investigación del ritmo en las lenguas naturales y sus variedades dialectales, es importante destacar que sí se tomará en cuenta el conocimiento cultural de los participantes, en la medida en que permita al investigador situar culturalmente el fenómeno en cuestión.

Asimismo, el sexto criterio se relaciona fuertemente con el quinto, en tanto se postula que los instrumentos deberían generarse in situ, como resultado de la observación y la indagación etnográfica. Este punto puede o no ser relevante para la investigación del ritmo en las lenguas naturales y sus variedades dialectales. Se estima que solo podrá ser relevante si los instrumentos se formulan a partir de una aproximación inicial, para, posteriormente, realizar otros acercamientos. En caso contrario, este principio no será adecuado, en tanto será necesario contar con instrumentos adaptados al estudio del fenómeno en cuestión, dado que no se mirará una diversidad de fenómenos etnográficos, sino que más bien el método etnográfico trabajará en paralelo con las mediciones de tipo cuantitativo. 
El séptimo criterio establecido por los autores aspira a tener siempre presente una perspectiva comparativa y transcultural; es decir, en una investigación de tipo etnográfico es necesario asumir tácitamente que la variación cultural es inherente a los fenómenos humanos. Respecto de este punto, es menester notar que fue el argumento de mayor peso a la hora de decidir incluir métodos etnográficos en la investigación. Esto, dado que se consideró que la variación del ritmo en las lenguas naturales y sus variedades dialectales es un fenómeno cultural e identitario y, por tanto, para su investigación se decidió adoptar no solo una metodología de investigación mixta, que integrase métodos cualitativos y cuantitativos, sino también un paradigma de corte tipológico, con el fin de observar el fenómeno en distintas comunidades de habla y sus respectivas variedades dialectales.

Los puntos octavo y noveno se relacionan con la disciplina per se. En el octavo punto, los autores arguyen que la etnografía tiene como misión hacer explícito lo implícito y lo tácito de los fenómenos que se observan. Respecto de esto, se considera que este principio es apropiado para el estudio del ritmo en las lenguas naturales y sus variedades dialectales, dado que se investigará el fenómeno del ritmo en las lenguas como un fenómeno situado, que si bien no es explícito en las comunidades, genera un sentido de pertenencia e identidad a una comunidad de habla específica.

Por su parte, el noveno punto indica que la etnografía debe promover el despliegue del conocimiento cultural de la forma más natural posible; dicho de otro modo, el investigador debe permitir que los informantes puedan hablar de manera fluida en las entrevistas que se realicen y será su deber no predeterminar las respuestas de sus informantes. Ahora bien, este criterio no es completamente adecuado para el estudio de las lenguas naturales y sus variedades dialectales. Si bien para recolectar los datos cualitativos se privilegiará la interacción y el despliegue del conocimiento cultural, en el caso de la recolección de los datos cuantitativos será necesario crear instrumentos de elicitación y tareas específicas para mediar y dirigir la interacción con los hablantes.

En cuanto a lo anterior, el décimo punto se refiere a los instrumentos. Se advierte que se deben preferir los aparatos o instrumentos que permitan recoger datos inmediatos, naturales y detallados, como lo son las cámaras, las grabaciones de audio y el video. Este punto es completamente pertinente para el estudio del ritmo en las variedades dialectales de las lenguas naturales. Tanto los métodos cualitativos como los cuantitativos utilizarán instrumentos, tales como grabadoras o cámaras de video. Ahora bien, es preciso destacar que en el caso de la recolección de los datos cuantitativos de esta investigación, lo más probable es que sea también necesario realizar algunas grabaciones en una cabina insonorizada, lo cual se contrapone con la situación de naturalidad que se busca generar con este tipo de instrumentos. 
Finalmente, y como criterio global, los autores indican que el etnógrafo debe situarse en el contexto dado mediante interacciones personales. De esta manera, su forma de reportar podrá ser más narrativa y cumplirá el objetivo de promover la coloquialidad y la fluidez en el intercambio con los participantes. A este respecto, conviene indicar que este punto es pertinente para la metodología cualitativa del estudio, dado que se buscará indagar acerca de conceptos como la identidad, la afiliación a un grupo por medio del ritmo de una variedad particular, etc.

A modo de conclusión, cabe hacer notar que muchos de los métodos enunciados anteriormente son pertinentes para el objeto de estudio en cuestión. Dada esta condición, a continuación se propondrá un diseño mixto, que integre tanto las mediciones cuantitativas mencionadas en el apartado 3 como los métodos etnográficos considerados relevantes en este apartado.

\section{PROPUESTA DE UN MODELO MIXTO PARA APROXIMARSE AL ESTUDIO DEL RITMO EN LAS LENGUAS NATURALES Y SUS VARIEDADES DIALECTALES}

En vista de la revisión que se realizó tanto de algunos métodos cuantitativos como cualitativos para el estudio del ritmo en las lenguas naturales y sus variedades dialectales, se propondrá un diseño mixto para el estudio del ritmo en las lenguas naturales desde una perspectiva social, descriptiva y tipológica. Antes de proceder a realizar la propuesta per se, se presentará una breve discusión sobre los beneficios de las metodologías mixtas para este tipo de investigaciones en particular.

A propósito de los distintos paradigmas para estudiar el lenguaje como fenómeno social, Angouri (2010: 30) asume una posición pragmática, que es también la línea que se seguirá en esta nota, en el sentido de considerar que las metodologías son una colección de técnicas y no estructuras de pensamiento saturadas ideológica y epistemológicamente. De este modo, se entenderán las técnicas y métodos cuantitativos y cualitativos como metodologías armónicas para un diseño de tipo mixto y no se cuestionarán sus incompatibilidades a nivel epistemológico, dado que no es el objeto de estudio de este artículo.

Ahora bien, y respecto de lo anterior, Angouri (2010) es enfática en señalar que el concebir los métodos de cada paradigma como una colección de técnicas no implica que su utilización conjunta sea un mero ejercicio de mixtura o superposición, desprovisto de lógica y rigor metodológico (31). En 
este sentido, la autora advierte que la responsabilidad de usar metodologías mixtas yace en el criterio de cada investigador, pues es el pesquisador quien deberá evaluar la pertinencia del uso de metodologías mixtas y, de así serlo, es él o ella quien elegirá qué métodos son apropiados para su objeto de estudio (Miles y Huberman 1994: 41 en Angouri: 32).

De esta manera, y basándose en Tashakkori y Creswell (2007: 4 en Angouri 2010: 33), la autora hace notar que existen diferentes tipos de metodologías mixtas, que pueden integrar ya sea uno o varios de los siguientes aspectos:

- Dos tipos de preguntas de investigación (desde una perspectiva cualitativa y cuantitativa);

- Formas distintas de desarrollar las preguntas de investigación (participativa versus planeadas con antelación);

- Dos tipos de muestreo (Ej.: probabilístico versus muestreo intencional);

- Dos tipos de recolección de datos (ej.: focus groups y encuestas)

- Dos tipos de datos (Ej.: númericos y textuales);

- Dos tipos de análisis de datos (Ej.: estadísticos y temáticos);

- Dos tipos de conclusiones (Ej.: emic y etic; objetivas y subjetivas).

Así también, Angouri (2010) indica que en los diseños mixtos la triangulación de los datos es un paso fundamental. A este respecto, menciona cuatro tipos de triangulación:

- Triangulación de los datos (aplicación de más de un método de muestreo en la recolección de los datos);

- Triangulación de investigadores (involucramiento de más de un investigador);

- Triangulación teórica (uso de más de una postura teórica);

- Triangulación metodológica (uso de más de una metodología) (Angouri, 2010: 34).

De acuerdo con Angouri (2010), la triangulación permite una mejor compresión de las preguntas de investigación y de los contextos situados. Por su parte, Dörnye (2007 en Angouri: 35) argumenta que mediante la triangulación de los datos se llega a una mejor compresión de los fenómenos, ya sea porque los datos convergen o divergen al momento de realizar las triangulaciones.

Pues bien, en vista de lo señalado anteriormente, a continuación se discutirá una propuesta de un diseño mixto para el estudio del ritmo en las lenguas naturales y sus variedades dialectales. En primer lugar, se 
establece que el diseño mixto para estudiar el ritmo en las lenguas naturales constará de dos preguntas de investigación: la primera indagará acerca del entroncamiento cultural del ritmo lingüístico, en tanto categoría identitaria y patrimonio cultural de comunidades de habla específicas. Por su parte, la segunda pregunta explorará patrones cuantitativos del ritmo en las variedades dialectales en relación con los índices propuestos por Ramus et al. (1999) y Low et al. (2000) respecto de la duración de los intervalos vocálicos, consonánticos, la duración total de las oraciones y el índice de PVI.

Para responder a estas preguntas de investigación, se realizarán dos tipos de muestreo. El primero consistirá en uno de tipo intencional, el cual tendrá como objetivo recolectar datos cualitativos sobre la identidad y el patrimonio asociado al ritmo en grupos específicos de la comunidad de habla a estudiar. Estos grupos se definirán in situ, en virtud de lo observado por el investigador durante sus primeros acercamientos etnográficos. Una vez efectuado este muestreo y habiéndose desarrollado una primera aproximación a la comunidad de habla, se procederá a realizar un muestreo de tipo probabilístico. Este muestreo estará destinado a la recolección de los datos cuantitativos para medir los índices anteriormente mencionados.

Asimismo, el diseño mixto que se propone constará de dos tipos de recolección de datos: por un lado, se recolectarán datos cualitativos con un instrumento etnográfico a definir luego de las primeras observaciones in situ. En cuanto a la recolección del corpus para las mediciones cuantitativas, este se obtendrá mediante entrevistas estructuradas, realizadas en una cabina insonorizada, con un instrumento de elicitación a definir.

En virtud de lo anterior, se obtendrán dos tipos de datos a partir de este diseño: por un lado, datos de tipo textual, que se recolectarán mediante instrumentos cualitativos y, por otro lado, datos de tipo numérico derivados de las mediciones antes mencionadas. Dado que se contará con dos tipos de datos, se realizarán también dos tipos de análisis: un análisis que apuntará a la descripción del ritmo de una lengua o variedad dialectal específica en relación con la identidad y el patrimonio cultural que esta representa para sus hablantes, y un análisis estadístico de tipo descriptivo e inferencial, con el fin de poder generalizar los datos obtenidos de una muestra específica a la población de la comunidad de habla.

Finalmente, las conclusiones también serán de dos tipos. En virtud de los datos cualitativos, se propondrán conclusiones en relación con la identidad, la vinculación del ritmo con el patrimonio cultural de los hablantes y la coconstrucción social de las variedades dialectales de cada lengua a nivel suprasegmental. Con respecto a los datos cuantitativos, se argumentarán conclusiones de tipo objetivo basadas en los estadísticos descriptivos e inferenciales y, además, se establecerán relaciones tipológicas con otras variedades dialectales del español y con otras lenguas naturales. 
En resumen, cabe señalar que, de acuerdo con Angouri (2010), se realizarán tres tipos de triangulaciones en este diseño mixto: por un lado, se hará una triangulación teórica; es decir, se utilizará más de una postura teórica para entender el fenómeno en cuestión, dado que por un lado, se entenderá como un fenómeno social situado, pero por otro, se comprenderá como un fenómeno fonológico de patrones lingüísticos. Así también, se efectuará una triangulación de tipo metodológico y, finalmente, se hará una triangulación de los datos obtenidos, pues se realizará más de un tipo de muestreo.

\section{CONCLUSIONES Y REFLEXIONES FINALES}

Tal como señala pragmáticamente Angouri (2010), los investigadores en el área de la lingüística no necesariamente están interesados en los debates epistemológicos que rodean los diferentes paradigmas en los que se enmarcan sus investigaciones, sino que más bien utilizan los diferentes métodos y técnicas a su disposición en virtud de preguntas de investigación específicas.

Pues bien, la presente nota se planteó como objetivo explicitar esta posición, pues se postuló un diseño mixto de investigación para un objeto de estudio que ha sido investigado en los últimos años de manera eminentemente cuantitativa (Dauer 1983; Ramus et al. 1999; Low et al. 2000, etc.). Pese a la tradición de investigación que subyace al objeto de estudio y la mirada epistemológica que esta misma tradición conlleva, se considera que el diseño mixto que se propone en este artículo es capaz de ampliar el alcance de este tipo de investigaciones, pues permite comprender el ritmo en las lenguas naturales y sus variedades dialectales no tan solo como un fenómeno cuantificable de naturaleza lingüística, sino también como una manifestación cultural situada y coconstruida, que se observa en comunidades de habla específicas.

Para finalizar, y como bien señalan Holmes y Meyerhoff (2003 en Angouri 2010), es claro que los investigadores combinan de manera fructífera diferentes aspectos de distintas metodologías con el objetivo de responder a las preguntas que se plantean y surgen en sus investigaciones. De este modo, se concluye que utilizar métodos etnográficos y mediciones cuantitativas entrega una visión más holística del fenómeno en cuestión, dado que permite extender el fenómeno más allá de su naturaleza lingüística, habilitando comprenderlo como un fenómeno cultural, vinculado al patrimonio y a la identidad de los sujetos. 


\section{REFERENCIAS BIBLIOGRÁFICAS}

ABERCROMBIE, DAVID. 1967. Elements of general phonetics (Vol. 203). Edinburgh: Edinburgh University Press.

Angouri, Jo. 2010. Quantitative, Qualitative or Both? Combining Methods in Linguistic Research. En en Lia Litosseliti, (ed.). Research methods in linguistics. Londres, Reino Unido: Continuum, pp. 29-48.

Dauer, Rebecca. 1983. Stress-timing and syllable-timing reanalyzed. Journal of Phonetics 11(1): 51-62. [en línea]. Disponible en http://psycnet.apa.org/psycinfo/1983-29886-001 [Consulta 10/10/2015].

De Pinho, José Ricardo Dordron. 2013. Considerações sobre o ritmo da fala no espanhol do Chile e da Espanha a partir do Índice de Variabilidade Pareada e de Coeficientes de Variação. Revista Leitura 2(52). [en línea]. Disponible en http://www.seer.ufal.br/ index. $\mathrm{php} /$ revistaleitura/article/ view/1475 [Consulta 10/10/2015].

Low Ee-Ling, Esther Grabe y Francis Nolan. 2000. Quantitative Characterizations of Speech Rhythm: Syllable-Timing in Singapore English. Language and speech 43(4): 377401. [en línea]. Disponible en http://las.sagepub.com/content/43/4/377.short [Consulta $10 / 10 / 2015]$.

NoKes, JACQUI Y JENNIFER HAYes. 2012. Acoustic correlates of rhythm in New Zealand English: A diachronic study. Language Variation and Change 24(1): 1-31. [en línea]. Disponible en http://journals.cambridge.org/action/displayAbstract?fromPage $=$ online \&aid $=852373$ 6\&fileId=S0954394512000051[Consulta 10/10/2015].

Pike, Kenneth L. 1945. The intonation of American English. Ann Arbor, EE.UU: University of Michigan Press.

Prieto, Pilar, Maria del Mar Vanrell, Lluïsa Astruc, Elinor Payne y Brechtje Post. 2012. Phonotactic and phrasal properties of speech rhythm. Evidence from Catalan, English, and Spanish. Speech Communication 54 (6): 681-702.

Ramus, Frank, Marina Nespor y Jacques Mehler. 1999. Correlates of linguistic rhythm in the speech signal. Cognition 73(3): 265-292. [en línea]. Disponible en http://www. sciencedirect.com/science/article/pii/S001002779900058X [Consulta 10/10/2015].

Roach, Peter. 1982. On the distinction between 'stress-timed'and 'syllable-timed'languages. En David Crystal (ed.). Linguistic controversies. Nueva York, EE.UU.: Academic Press, pp. 73-79.

Rodríguez Gómez, Gregorio, JaVier Gil Flores y EduARdo García Jiménez. 1996. Metodología de la investigación cualitativa. Málaga: Aljibe.

SeALEy, Alison. 2007. Linguistic ethnography in realist perspective. Journal of Sociolinguistics 11(5): 641-660. [en línea]. Disponible en http://onlinelibrary.wiley.com/doi/10.1111/ j.1467-9841.2007.00344.x/abstract [Consulta 10/10/2015].

Tan, Rachel Siew Kuang y Ee-Ling Low. 2014. Rhythmic Patterning in Malaysian and Singapore English. Language and Speech 57(2): 196-214. Disponible en http://las.sagepub. com/content/57/2/196.short [Consulta 10/10/2015].

Toledo, Guillermo. 2010. Métricas rítmicas en tres dialectos Amper-España. Estudios filológicos 45: 93-110. Disponible en http://www.scielo.cl/scielo.php?pid=S007117132010000100008\&script=sci_arttext [Consulta 10/10/2015].

White, Laurence y Sven L. Mattys. 2007. Calibrating rhythm: First language and second language studies. Journal of Phonetics 35(4): 501-522. 
